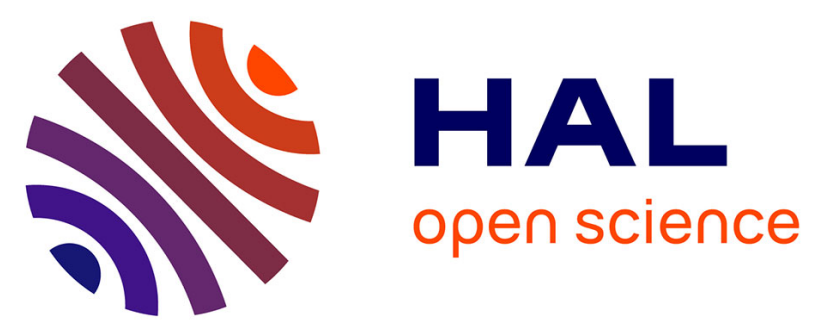

\title{
2D thermo-mechanical contact simulations in a functionally graded material: a multigrid-based approach
}

Benoît Watremetz, Marie-Christine Baietto, Antonius Lubrecht

\section{- To cite this version:}

Benoît Watremetz, Marie-Christine Baietto, Antonius Lubrecht. 2D thermo-mechanical contact simulations in a functionally graded material: a multigrid-based approach. Tribology International, 2007, 40 (5), pp.754-762. 10.1016/j.triboint.2006.07.001 . hal-01951573

\section{HAL Id: hal-01951573 \\ https://hal.science/hal-01951573}

Submitted on 7 Jul 2021

HAL is a multi-disciplinary open access archive for the deposit and dissemination of scientific research documents, whether they are published or not. The documents may come from teaching and research institutions in France or abroad, or from public or private research centers.
L'archive ouverte pluridisciplinaire HAL, est destinée au dépôt et à la diffusion de documents scientifiques de niveau recherche, publiés ou non, émanant des établissements d'enseignement et de recherche français ou étrangers, des laboratoires publics ou privés.

\section{(ㄷ)(1) $\$$}

Distributed under a Creative Commons Attribution - NonCommerciall 4.0 International 


\title{
2D thermo-mechanical contact simulations in a functionally graded material: A multigrid-based approach
}

\author{
B. Watremetz*, M.C. Baietto-Dubourg, A.A. Lubrecht \\ LaMCoS, Domaine scientifique de la Doua, Batiment Jean d'Alembert, 18-20 rue des sciences, 69621 Villeurbanne CEDEX, France
}

\begin{abstract}
The development and selection of coatings or coating combinations is a complex and costly task. Numerical simulations provide a great help to analyze the behavior of coatings and layer interfaces under mechanical and thermal loading through the computation of stress and strain fields. They are also used to fine tune the geometrical configuration and define optimal thermo-mechanical properties related to the applied stress to enhance wear and crack resistance. Coating design has been based mainly on discrete layer models with abruptly changing properties at interface perfectly bonded to a substrate. These assumptions result in discontinuities in stress and temperature fields at the interface between successive layers. These models are based either on integral transform (classically Fourier transform) or finite element (FE) methods. The former cannot handle 3D thermo-mechanical problems. The latter demands huge calculation times, especially when considering thin layers with very small elements. The aim of this paper is to account for materials with continuously changing properties and to obtain an improved prediction of the resistance of such graded materials. This gradation may be linked to surface treatments like nitruration, thermal treatment, .. . or to the deposition of coatings inducing diffuse boundaries between those dissimilar materials. The proposed model aims at providing valuable description and understanding of the behavior and resistance of such graded material. It is based on a second order finite difference (FD) formulation of the continuous thermal and elasticity equations. It can handle any kind of depth dependence of the material properties. Multigrid techniques are implemented to accelerate the convergence, reduce CPU time and thus permit the use of fine grids to accurately describe the variation in the material properties. The first step of this project consists in implementing the above-cited numerical techniques in a 2D plane strain model.
\end{abstract}

Keywords: FGM; Coating; Thermo-mechanic; Simulation; Multigrid

\section{Introduction}

Coatings or coating combinations are widely used to increase life and operational performance of engineering materials. Tribology is one of the major application fields of coatings as the properties of contacting bodies can be markedly improved by coating them with soft or hard layers according to the application.

When coating and substrate are elastically dissimilar, the contact stresses may differ from those generated in the uncoated case. The actual stress distribution can only be

This paper was presented at the 32nd Leeds-Lyon Symposium in Lyon, 6-9 September 2005.

${ }^{*}$ Corresponding author. Tel.: + 33472438980 ; fax: +33472438500 .

E-mail address: benoit.watremetz@insa-lyon.fr (B. Watremetz). calculated by numerical or semi-numerical techniques for layered materials. The numerical simulation of thermomechanical deformations of elastic bodies becomes very difficult when thin coatings with varying properties are involved. This topic has been the subject of several studies. The models usually consider discrete isotropic layers (Fig. 1(a)) with constant properties and a sharp transition at the interface. Furthermore, perfect bounds at interfaces are assumed. It leads to inherent stress discontinuities at interface. These methods are generally based either on integral transform combined with fast Fourier transform (FFT) algorithm or on finite element (FE) methods.

$\mathrm{Ju}$ and Liu [1] and Leroy et al. [2] used the Fourier transform to study the thermo-mechanical deformation of a 2D layered elastic half space subjected to a moving heat source. A 3D mechanical study was performed by Plumet 


\section{Nomenclature}

$E_{\mathrm{c}} \quad$ Young's modulus of the coating

$E_{\mathrm{s}} \quad$ Young's modulus of the substrate

$f \quad$ PDE right-hand side

$h \quad$ grid spacing

$H_{\mathrm{c}} \quad$ coating thickness

$i \quad$ line number

$j \quad$ column number

$k \quad$ thermal conductivity

$N_{x}, N_{y}$ number of nodes along $x$ and $y$ directions

$q \quad$ thermal flow

[3] using the same techniques. However, applying FFT to such problems introduces a numerical error, which will be referred to as the periodicity error. This error can be avoided by extending the surface grid sufficiently far beyond the contact area. The associated increase in the number of grid nodes significantly degrades the computational efficiency of the FFT-based method. Several techniques have been introduced to overcome this degradation: Polonsky and Keer [4,5] counterbalanced the periodicity error by using a special correction term, which is computed using a variation of the MLMS technique [6]. Nogi and Kato [7] combined the conjugate gradient method with the FFT technique for solving the rough contact problem for both homogeneous and layered solids. Liu et al. $[8,9]$ extended the FFT method in the light of the discrete convolution (DC) theorem. The DC method associated with the processes of zero padding and wrap around order can completely avoid the error associated with FFT at a cost of only doubling the domain dimensions. This approach is named DC-FFT algorithm. However, FFT techniques cannot handle 3D thermo-

$\begin{array}{ll}T & \text { temperature } \\ u, v & \text { displacements along } x, y \text {-axis } \\ L & \text { PDE operator } \\ \lambda, \mu & \text { Lamé's coefficients } \\ \alpha & \text { thermal expansion coefficient } \\ \sigma_{x x} & \text { tensile stress } \\ \sigma_{x y} & \text { shear stress } \\ v_{1}, v_{2} & \text { number of relaxation sweeps }\end{array}$

Indice

$h \quad$ level corresponding to $h$ mesh size

mechanical problems, as the corresponding equations do not verify the assumption of the biharmonic displacements operator.

Analytical methods combined with asymptotic analysis, assuming the coating as a bond between two contacting bodies can also be used. In these methods, the Lamé coefficients are set proportional to the height $\varepsilon$ of the joint. Bayada et al. [10] studied the behavior of three bodies, one of them sliding on a thin layer, itself bonded to a rigid support. The characteristic feature of their study is the nonlinear Tresca law used to describe the contact between the two elastic bodies. This method is essentially developed for elastic bodies and thin layers.

3D thermo-mechanical simulations are mainly performed using FE techniques [11]. The main disadvantage of this approach for thin layers is the large system of equations arising from the fine mesh. Conventional numerical methods take unacceptably long times even on modern computers. The computation speed can be increased by using advanced numerical techniques such as multigrid methods.

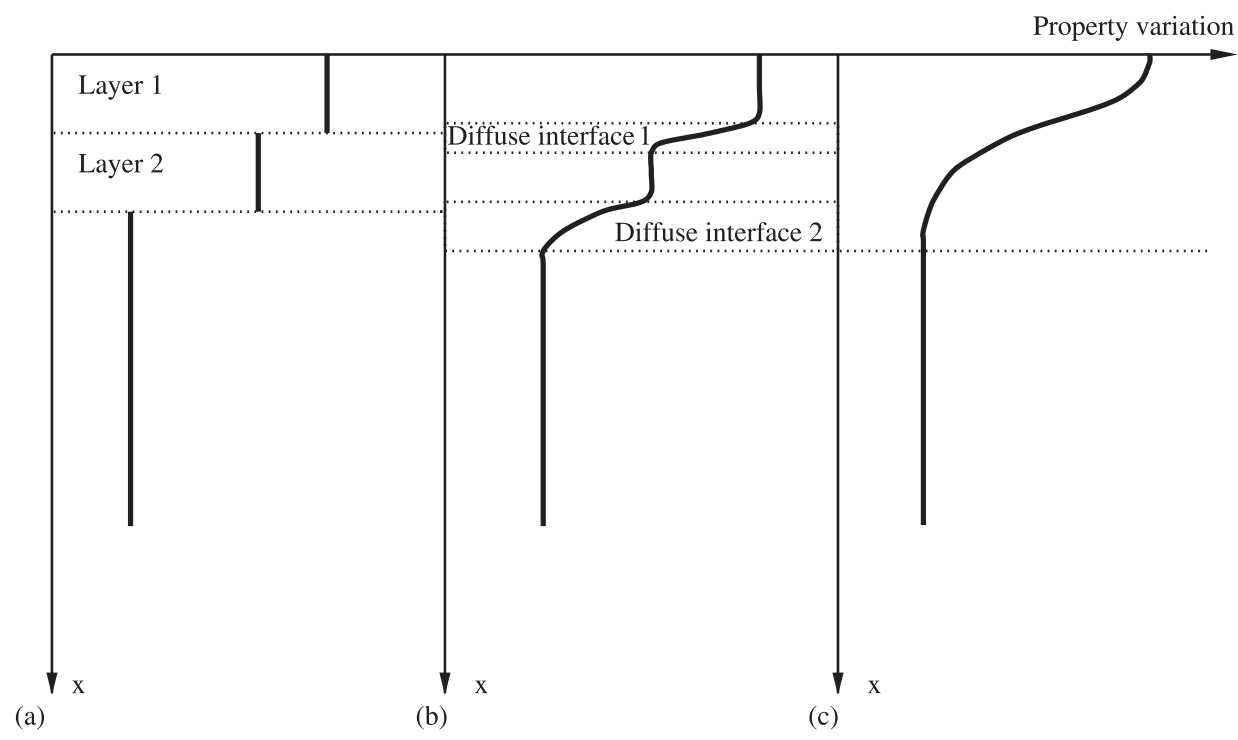

Fig. 1. Property variation with depth: (a) former models: sharp changing properties at interface; (b) continuously changing properties across diffuse boundaries; and (c) continuously graded material. 
The present work is a first step toward a complete $3 \mathrm{D}$ thermo-mechanical model encompassing functionally graded materials (FGM) and coating/substrate systems. The mechanical and thermal properties of the system vary continuously with depth. This gradation includes: (i) a progressive change in properties (linear, parabolic, exponential, ...) corresponding for instance to a treatment like nitruration, thermal treatment (Fig. 1(c)), (ii) a continuous but steep variation across a thin diffuse boundary between two dissimilar materials corresponding to a composition or structural transition (Fig. 1(b)) and (iii) any combination of the two previous possibilities. The intention is to understand and evaluate the temperature, the stress and strain fields in the system. The perfect bonding assumption used in former models is intrinsically contained in this new formulation. Smooth graded stress variations across diffuse boundaries are obtained.

The model is presented in the first section. The effects on the convergence speed of the property mismatch and the coating thickness are investigated in the second section in the case of a coating with a thin diffuse boundary over a substrate. Finally, an application devoted to thin coatings with a thicker diffuse boundary (Fig. 1(b)) is presented. Hereafter the boundary and the layer with varying properties is simply called coating.

\section{Theoretical background}

The model can be split into two parts as small strain assumptions are retained: the thermal and the elastic one. The thermal part of the solution process is first computed independently of the elastic deformations. Then the elasticity equations are solved accounting for the temperature gradient.

\subsection{Thermal model}

The differential equation of heat conduction for a stationary, homogeneous, isotropic solid with constant thermal properties and no heat generation reads (Fourier conduction equation) [12]:

$\nabla(\mathbf{k} \nabla T)=\frac{\mathbf{k}}{\alpha} \frac{\partial T}{\partial t}$,

where $\alpha=k / \rho C_{p}$ is the thermal diffusivity.

For the case of a coating-substrate system, the gradation of the properties is supposed to be only a function of depth. A reduced form of Eq. (1) is obtained with thermal properties function of $x$ and independent of the temperature:

$\frac{\partial}{\partial x}\left(k_{x} \frac{\partial T}{\partial x}\right)+k_{y} \frac{\partial^{2} T}{\partial y^{2}}=\frac{\mathbf{k}}{\alpha} \frac{\partial T}{\partial t}$.

\subsection{Thermal boundary conditions (BC)}

Finally, $\mathrm{BC}$ in terms of prescribed temperature derivative (Neumann BC) or temperature (Dirichlet BC) are con- sidered on each side of the domain. On the surface boundary the heat flux is prescribed:

$q_{0}(x=0, y)=-\left.k(0) \frac{\partial T}{\partial x}\right|_{x=0}$

while on the other boundaries the temperature is imposed.

\subsection{Mechanical model}

The constitutive equations of a $2 \mathrm{D}$ elasticity problem can be reduced to two partial differential equations (PDE) written in terms of displacements (three in the general 3D case). To obtain these equations, the elastic stress-strain relations are substituted into the equilibrium equations. This leads to the classical Navier equations of elasticity in a continuous medium [13].

In the case of an elastically graded coated system, the elastic coefficients $E$ and $v$ are no longer constant. The equilibrium equations, accounting for the anisotropic properties $E(x)$ and $v(x)$, are the generalized Navier's equations:

$$
\begin{gathered}
\frac{\partial}{\partial x_{j}}\left(\mu \frac{\partial u_{i}}{\partial x_{j}}\right)+\frac{\partial}{\partial x_{j}}\left(\mu \frac{\partial u_{j}}{\partial x_{i}}\right)+\frac{\partial}{\partial x_{i}}\left(\lambda \frac{\partial u_{j}}{\partial x_{j}}\right) \\
=\frac{\partial}{\partial x_{i}}((3 \lambda+2 \mu) \alpha T), \quad i=1,2,
\end{gathered}
$$

$$
\lambda=\frac{E v}{(1+v)(1-2 v)}, \quad \mu=\frac{E}{2(1+2 v)}
$$

with $\lambda=\lambda(x)$ and $\mu=\mu(x)$ the Lamé's coefficients.

The Young's modulus $E$ and Poisson's ratio $v$ can be any function of depth.

Linear strain, small displacement and plane strain assumptions are retained leading to (4):

$$
\begin{aligned}
& \frac{\partial}{\partial x}\left((\lambda+2 \mu) \frac{\partial u}{\partial x}\right)+\mu \frac{\partial^{2} u}{\partial y^{2}}+\frac{\partial}{\partial x}\left(\lambda \frac{\partial v}{\partial y}\right)+\mu \frac{\partial^{2} v}{\partial x \partial y} \\
& \quad=\frac{\partial}{\partial x}((3 \lambda+2 \mu) \alpha T), \\
& (\lambda+2 \mu) \frac{\partial^{2} v}{\partial y^{2}}+\frac{\partial}{\partial x}\left(\mu \frac{\partial u}{\partial y}\right)+\frac{\partial}{\partial x}\left(\mu \frac{\partial v}{\partial x}\right)+\lambda \frac{\partial^{2} u}{\partial x \partial y} \\
& =\frac{\partial}{\partial y}((3 \lambda+2 \mu) \alpha T) .
\end{aligned}
$$

\subsection{Mechanical BC}

System (6) is completed with the BC; both Dirichlet and Neumann BC can be imposed (Fig. 2). Stress-based BC are expressed in terms of displacements and strains using the stress-displacement relations:

$$
\begin{aligned}
\sigma_{x x} & =(\lambda+2 \mu) \frac{\partial u}{\partial x}+\lambda \frac{\partial v}{\partial y}-(3 \lambda+2 \mu) \alpha T \\
\sigma_{x y} & =\mu\left(\frac{\partial u}{\partial y}+\frac{\partial v}{\partial x}\right) .
\end{aligned}
$$




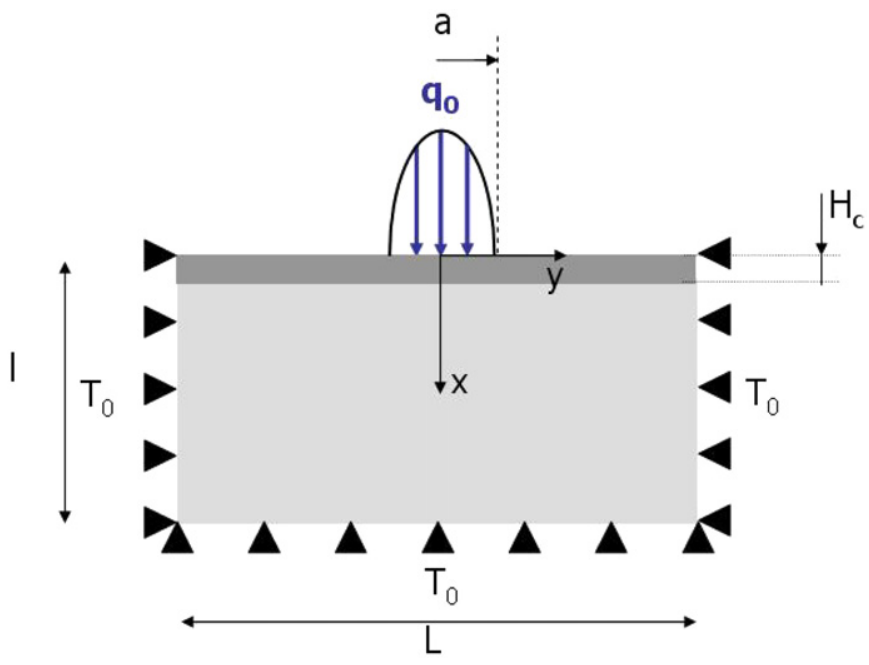

Fig. 2. Thermal and mechanical boundary conditions.

\section{Numerical treatment}

Eqs. (2) and (6) are discretized using a second order central finite difference (FD) scheme on a uniform grid. Very dense grids can be generated and hence large systems of equations have to be solved. The classical solution techniques using Gauss-Seidel relaxation require large memories and huge computing times. Multigrids methods are known to accelerate the convergence of such large problems.

\subsection{Multigrid techniques}

Conventional iterative methods converge slowly as the long wavelength error present in the solution slowly diminishes with successive relaxation sweeps. Multigrid techniques overcome this difficulty by using a sequence of grids. The numerical solution of a $1 \mathrm{D}$ elliptic equation on a uniform grid of size $N$ is thus performed with new coarser grids of size $(N / 2),(N / 4), \ldots$ defining successive levels. A $V\left(v_{1}, v_{2}\right)$-cycle consists of $v_{1}$ relaxation sweeps on each level combining restrictions (from the finest to the coarsest grid), the exact numerical solution at the coarsest level and the prolongation from the coarsest to the finest grid with $v_{2}$ relaxation sweeps on each level. The grids communicate with one another through restriction and prolongation operators $[14,15]$. A $V\left(v_{1}, v_{2}\right)$-cycle, with $v_{1}=v_{2}=2$ is used for the thermal problem and $v_{1}=v_{2}=5$ for the elasticity problem. More relaxations do not enhance the efficiency of the relaxation process. The residual used in this section as an error indicator is calculated for each level as follows [17]:

$\operatorname{res}^{h}=\sum_{i, j}\left|\frac{L^{h} u^{h}(i, j)-f^{h}(i, j)}{N_{x}^{h} N_{y}^{h}}\right|$.

Efficient multigrid algorithms for the solution of PDE require good ellipticity, which implies that non-smooth error components can be solved by local processing [16]. The elasticity and thermal equations are elliptic by nature and a good multigrid efficiency might be expected. Unfortunately, while the treatment of the Dirichlet BC is straight forward, Neumann BC and the anisotropy of the problem rapidly degrade the solver efficiency. These points are detailed in the next sections.

\subsection{Boundary conditions}

Some difficulties in solving the elastic problem arise from the Neumann BC. In order to obtain a multigrid solver with the same efficiency as the one employing Dirichlet conditions, the $\mathrm{BC}$ have to fulfill two criteria:

- the consistency on the various grids,

- no introduction of local errors.

Venner et al. [17] relax the interior problem on the surface using the Neumann condition to fulfill both criteria. This method is well-suited for the thermal problem but the optimal convergence speed is not reached for the resolution of system (6) using (7) as shown in Fig. 3. Some prerelaxations are necessary to decrease the residual near boundaries before solving the entire problem, as described by Brandt [18].

\subsection{Anisotropy}

One key to the multigrid convergence rate is the consistency of the system on coarse and fine grids. The coarse grid operator must correctly approximate the fine grid operator.

Anisotropy arising from the variation of the properties within the material depth rapidly degrades the convergence rate. The two reasons are:

- around the substrate/coating diffuse boundary, the equations are losing their elliptic properties. The sharper the gradient, the more difficult the convergence;

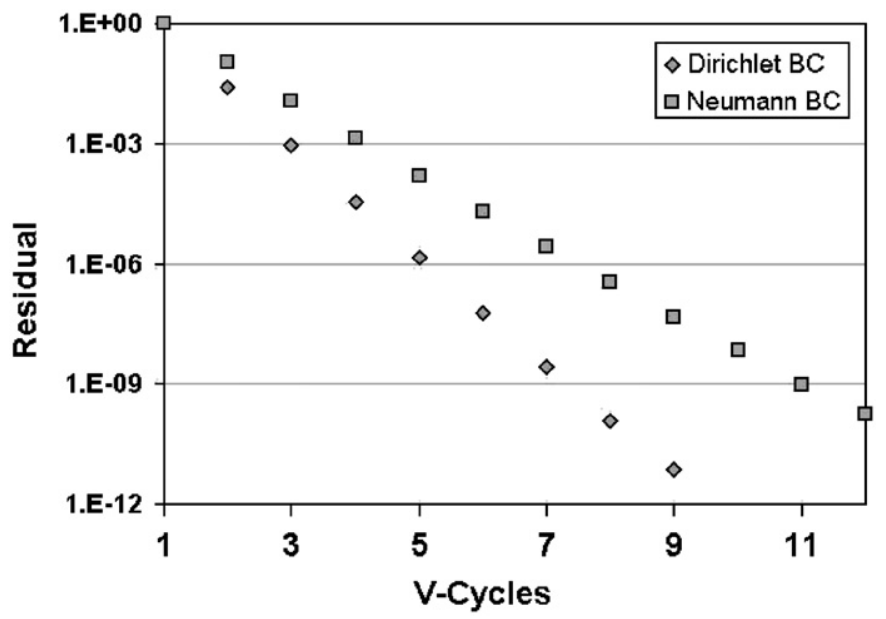

Fig. 3. Residual on finest level for Dirichlet and Neumann BC. 
- when the diffuse boundary is located in the vicinity of the free surface, it becomes numerically difficult to correctly capture the coating itself on coarse grids.

The influence of these two points on the convergence rate are illustrated hereafter.

The current reference problem is a pointwise, unit load applied on the free surface of a $3 * 3 \mathrm{~mm}$ coated elastic solid clamped on the three other boundaries. Thermal effects are not taken into account. A stepwise but continuous gradation in the mechanical properties (Fig. 1b) is considered. The thickness of the diffuse boundary is equal to one mesh size on the finest grid. This kind of gradation is the worse case from the numerical point of view. Calculations are performed with $512 * 512$ elements on the finest grid using 7 levels $(8 * 8$ elements on coarsest grid). The reference problem will be considered for all the numerical tests presented hereafter.

\subsection{Surface-interface interaction}

The intention is to evaluate the influence of the coating thickness $\left(H_{\mathrm{c}}\right)$ on the convergence rate of the elastic problem. No thermal effects are taken into account. The Young's modulus of the coating is six times higher than the substrate modulus $\left(E_{\mathrm{c}}=200 \mathrm{GPa}, E_{\mathrm{s}}=1200 \mathrm{GPa}\right.$, $\left.v_{\mathrm{c}}=v_{\mathrm{s}}=0.3\right)$ defining a ratio $E_{\mathrm{c}} / E_{\mathrm{s}}=\frac{1}{6} . H_{\mathrm{c}}$ is varying from $30 \mu \mathrm{m}$ (5 points on the finest grid) to $1 \mathrm{~mm}(150$ points). The residual variations are plotted in Fig. 4 for different coating thicknesses.

It is obvious that the number of nodes within the coating decreases as its thickness diminishes for a given mesh size. The coarse grids hence cannot correctly capture the coating properties as the number of nodes rapidly drops to 0 . Finally, the numerical problems solved on fine and coarse grids are not equivalent. The thinner the coating, the worse the convergence becomes. An alternative is to explicitly

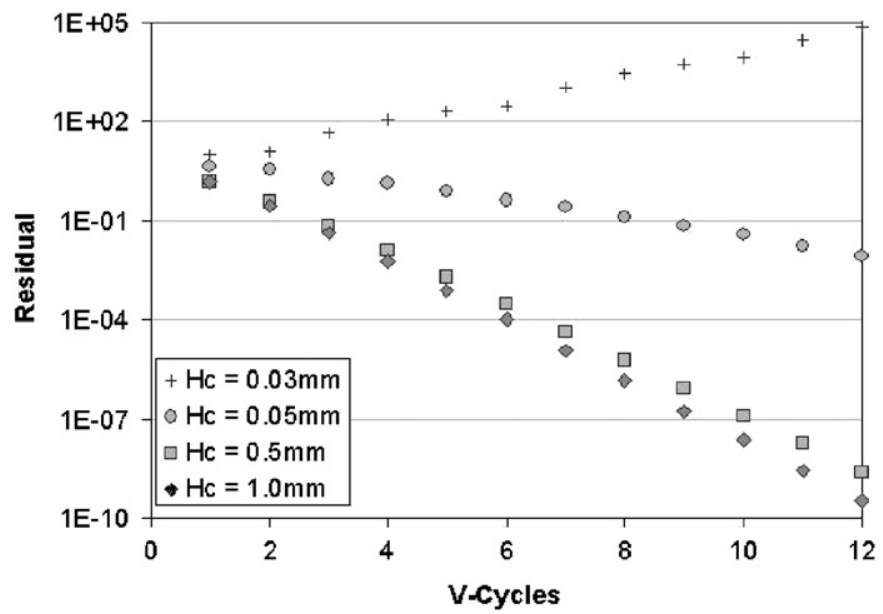

Fig. 4. Residual on finest level as a function of coating thickness with $E_{\mathrm{c}} / E_{\mathrm{s}}=\frac{1}{6}$. average the elastic ( $\lambda$ et $\mu$ ) and thermal $(k$ et $\alpha$ ) properties using full weighting techniques [17]. The main goal is to capture the information related to the property variation in depth and to solve an equivalent problem on any grid level. This is illustrated in Figs. 5 and 6. Fig. 5 shows the initial variation of $\lambda / \lambda_{\mathrm{s}}$ parameter for a fine and a coarse grid. On the coarse grid, the ratio varies from the value at the surface $\left(\lambda_{\mathrm{c}} / \lambda_{\mathrm{s}}\right)$ to the value in the substrate (1) over one mesh step. Fig. 6 shows the solution used to overcome the convergence difficulties of the solution method: the property variation is smeared around the interface. The problem is equivalent on the coarse and the fine mesh when using the averaging technique.

Fig. 7 shows the residual evolution with averaged properties. The variation of the Lamé coefficient with depth is plotted in Figs. 5 and 6. The convergence is improved by several orders of magnitude, even in the case

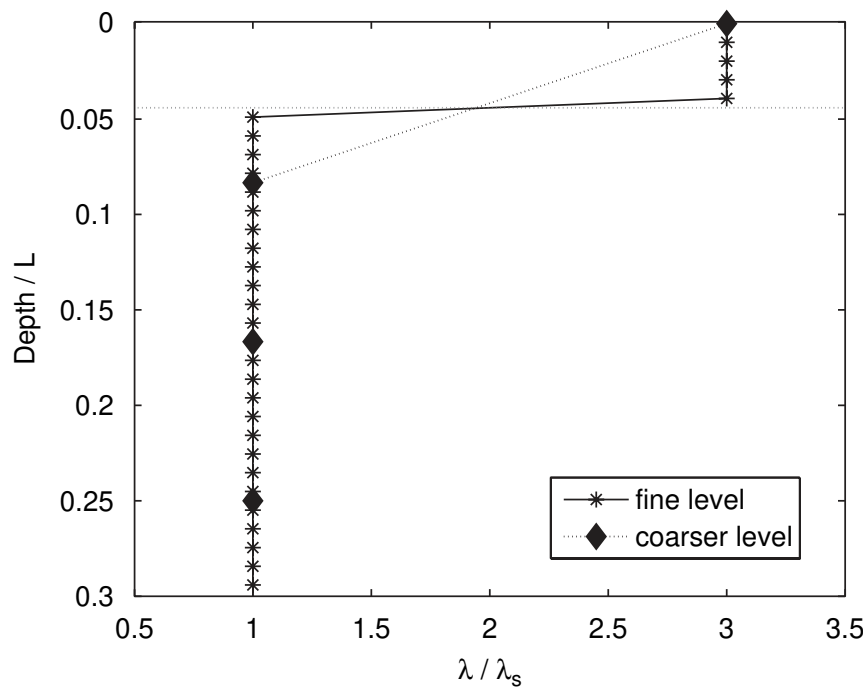

Fig. 5. Initial $\lambda / \lambda_{\mathrm{s}}$ parameter variation on a fine and a coarse mesh.

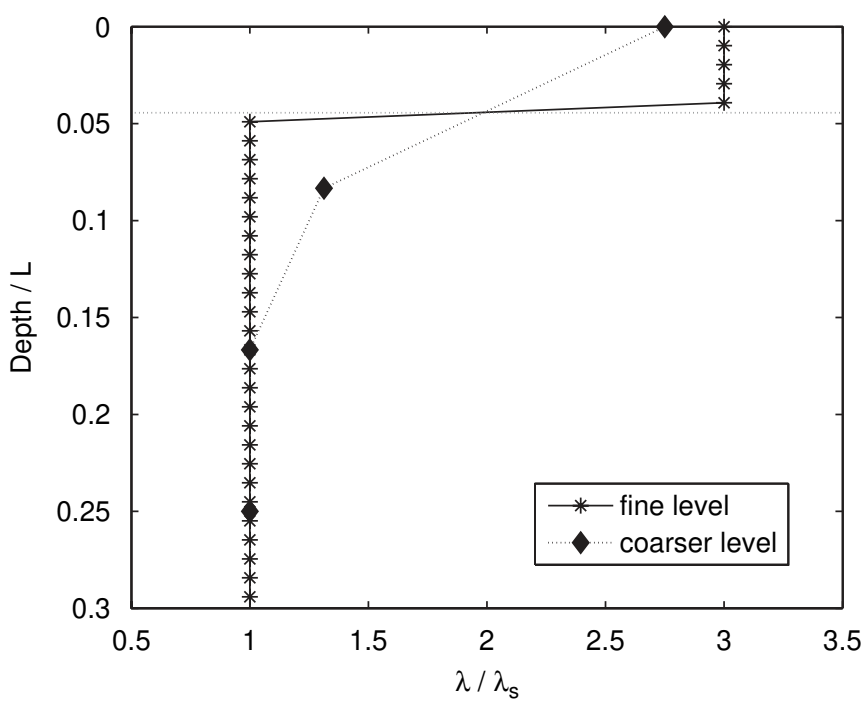

Fig. 6. $\lambda / \lambda_{\mathrm{s}}$ parameter variation on a fine and a coarse mesh with full weighting restriction. 


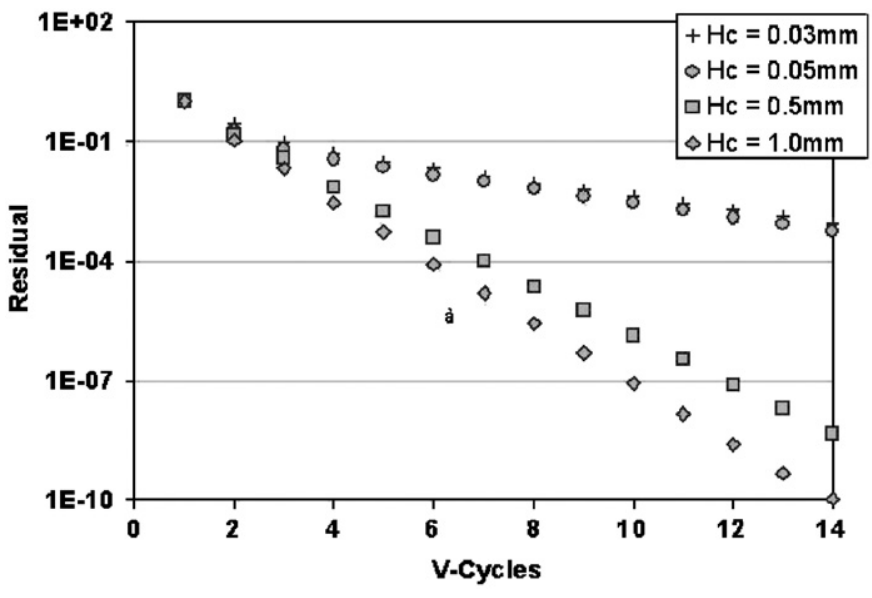

Fig. 7. Residual on finest level as a function of coating thickness with averaged properties $-E_{\mathrm{c}} / E_{\mathrm{s}}=\frac{1}{6}$.

$H_{\mathrm{c}}=1 \mathrm{~mm}$ for which the convergence was satisfactory. But above all convergence is obtained for the case $H_{\mathrm{c}}=$ $30 \mu \mathrm{m}$ while it previously diverged.

\subsection{Ellipticity}

The second difficulty arising from the anisotropy of the system is the loss of ellipticity due to the property gradient. This phenomenon can be observed from the stencil. As an example, the stencil $L_{1}^{h}$ of the first equation of system (6) is written:

$\frac{1}{h^{2}}\left[\begin{array}{c}Q_{i-1, j} \\ \mu_{i, j-1}-\left(Q_{i+1, j}+Q_{i-1, j}+2 \mu_{i, j}\right) \mu_{i, j+1} \\ Q_{i+1, j}\end{array}\right]$

with $Q=\lambda+2 \mu$.

The stencil becomes less symmetric as the property mismatch increases (Eq. (9)). This distortion in the stencil degrades the convergence speed. The reference problem has been defined above. The coating is $0.5 \mathrm{~mm}$ thick to avoid the interface/surface interaction discussed just above and focus on the ellipticity influence. The residual variation are plotted in Fig. 8 for $E_{\mathrm{c}} / E_{\mathrm{s}}$ ratios varying from 0.25 to 10 . The convergence rate is close to optimal for soft coatings $\left(E_{\mathrm{c}} / E_{\mathrm{s}}<1\right)$, good as long as $E_{\mathrm{c}} / E_{\mathrm{s}}<7$ but degrades for larger ratios. This problem can be overcome by performing line relaxation.

\subsection{Mesh refinement}

Coated systems are multiscale problems as a large difference between coating thicknesses, loaded zone size and the substrate dimensions exists. Therefore, a high grid resolution is required around the coating and the interface while elsewhere such level of refinement is not required, especially when considering a semi-infinite solid (solid size $\gg$ coating thickness and/or contact width). The MG technique is ideally suited for local mesh refinement. In

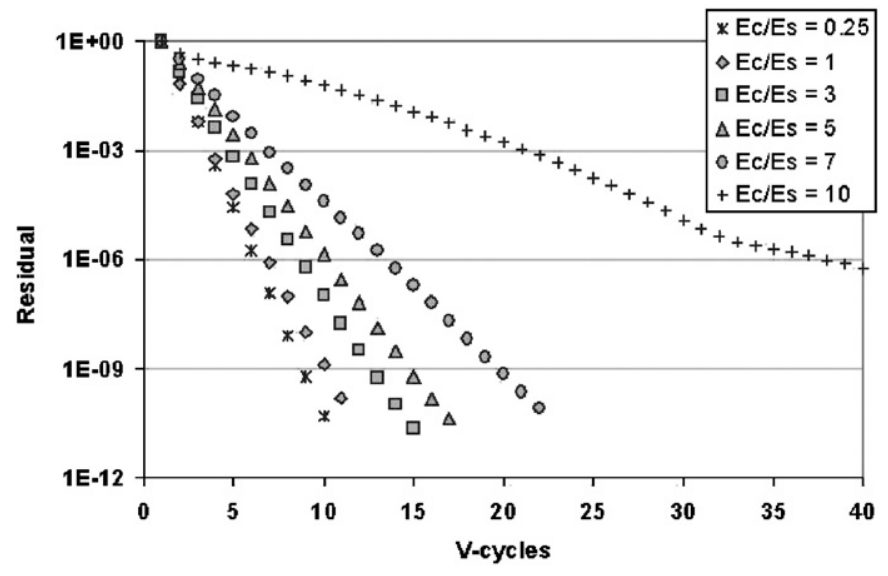

Fig. 8. Residual on finest level as a function of $E_{\mathrm{c}} / E_{\mathrm{s}}$ ratio with $H_{\mathrm{c}}=0.5 \mathrm{~mm}$.

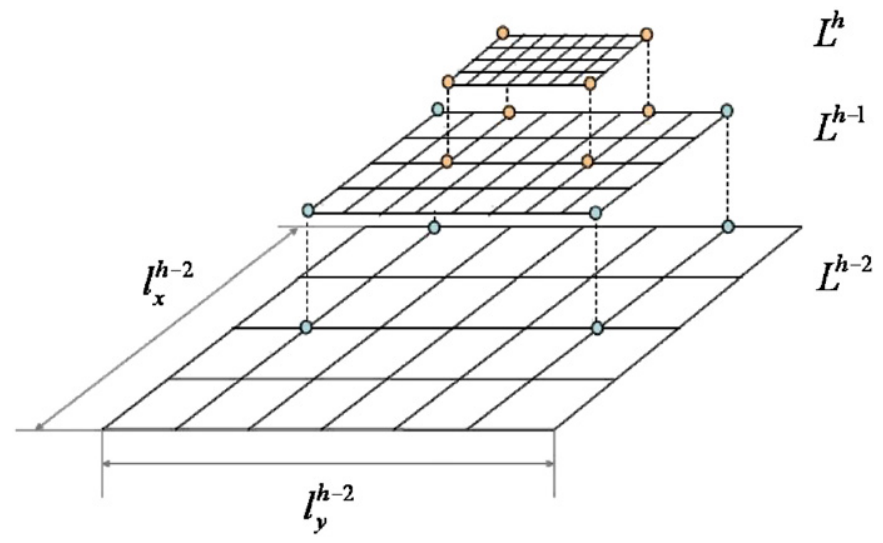

Fig. 9. Local refinement technique.

such cases, the finest grids can be restricted to smaller and smaller subdomains, whereas the coarse grids cover the entire domain. The resulting grid strongly looks like a nonequidistant grid, whilst keeping the advantages of an equidistant grid.

The refinement strategy consists in defining successive subdomains corresponding to successive levels where grid refinement is performed only locally (Fig. 9). The fine grid residuals and the coarse grid correction are only carried out in the local part where the fine grid exists [19].

The reference solution $u_{\text {ref }}$ based on classical solution over the whole domain is obtained using a $1024 \times 1024$ fine grid using 9 levels. The results obtained for three cases corresponding to three local refinement strategies $u_{\text {app }}$ are compared to the classical results in terms of error (two norms are defined by (10) [17] and computational cost. Data given in Table 1 corresponds to the zone covered by the different levels.

The domain covers $0 \leqslant x \leqslant 3.0$ and $-1.5 \leqslant y \leqslant 1.5$. In case 1 , only the finest level (9) is locally refined. In case 2 , both levels 9 and 8 are refined and in case 3, level 7 is also refined.

Fig. 10 illustrates the effect of local refinement on the residual evolution. The coating is $30 \mu \mathrm{m}$ thick using $E_{\mathrm{c}}=$ 
Table 1

Refinement data

\begin{tabular}{lllll}
\hline & Case & 1 & 2 & 3 \\
\hline Lev. 9 & $x$ & {$[0 ; 2]$} & {$[0 ; 1]$} & {$[0 ; 0.5]$} \\
& $y$ & {$[-1 ; 1]$} & {$[-0.5 ; 0.5]$} & {$[-0.25 ; 0.25]$} \\
Lev. 8 & $x$ & {$[0 ; 3]$} & {$[0 ; 2]$} & {$[0 ; 1]$} \\
& $y$ & {$[-1.5 ; 1.5]$} & {$[-1 ; 1]$} & {$[-0.5 ; 0.5]$} \\
Lev. 7 & $x$ & {$[0 ; 3]$} & {$[0 ; 3]$} & {$[0 ; 2]$} \\
& $y$ & {$[-1.5 ; 1.5]$} & {$[-1.5 ; 1.5]$} & {$[-1 ; 1]$} \\
\hline
\end{tabular}

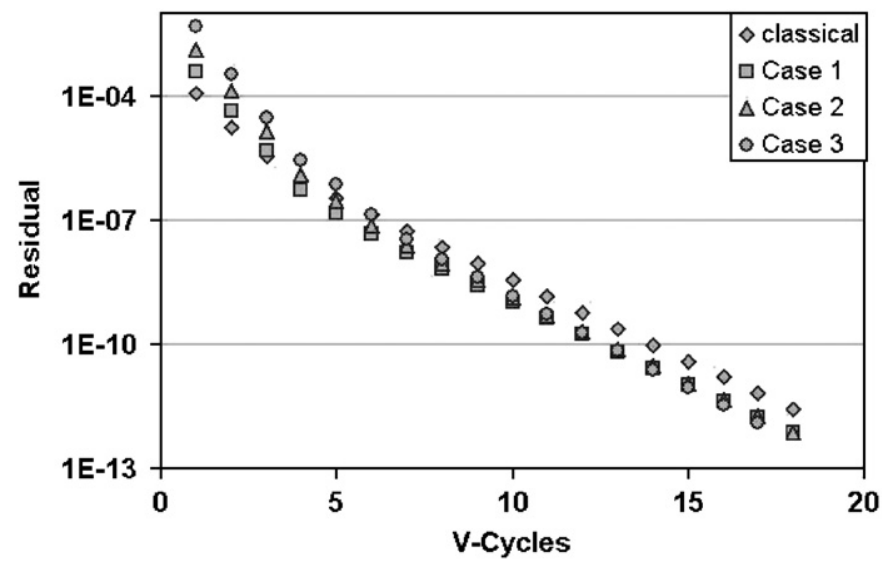

Fig. 10. Residual with three refinement strategies.

Table 2

Refinement error, CPU ratio

\begin{tabular}{llll}
\hline Number of refined level & 1 & 2 & 3 \\
\hline$\left\|u_{\text {ref }}-u_{\text {app }}\right\|_{\infty}$ & $3.93 \times 10^{-4}$ & $4.50 \times 10^{-4}$ & $8.13 \times 10^{-4}$ \\
$\left\|u_{\text {ref }}-u_{\text {app }}\right\|$ & $1.24 \times 10^{-4}$ & $2.74 \times 10^{-4}$ & $9.76 \times 10^{-5}$ \\
CPU ratio & 0.37 & 0.12 & 0.043 \\
\hline
\end{tabular}

$800 \mathrm{GPa}$ and $E_{\mathrm{s}}=200 \mathrm{GPa}$ and $v_{\mathrm{c}}=v_{\mathrm{s}}=0.3$. The local refinement solutions obtained on the fine grid are very similar to the reference solution.

$$
\begin{aligned}
& \left\|u_{\mathrm{ref}}-u_{\mathrm{app}}\right\|=\sum_{i, j} \frac{\left\|u_{\mathrm{ref}}-u_{\mathrm{app}}\right\|}{N^{2} u_{\mathrm{ref}}}, \\
& \left\|u_{\mathrm{ref}}-u_{\mathrm{app}}\right\|_{\infty}=\max \left(\left\|\frac{u_{\mathrm{ref}}-u_{\mathrm{app}}}{u_{\mathrm{ref}}}\right\|\right) .
\end{aligned}
$$

Table 2 shows the difference between the classical solution and the one with grid local refinements. $u_{\text {ref }}$ is the reference solution, $u_{\text {app }}$ the approximate solution on the highest level with mesh refinement. CPU ratio is the ratio between the solution times with and without mesh refinement.

The first norm quantifies the maximum of the error, as the second one shows the mean error. The maximum increases as the refinement reaches lower levels, but the quality of the solution is still very good. The calculation time shows that the saving in terms of computational cost is considerable. The problem can be solved with almost the same accuracy with a 20 times smaller computational effort. The numerical error is $8 \times 10^{-5}$ between levels 9 and 10 using the full domain. It is $10^{-4}$ with local mesh refinement.

\section{Application}

In this section, a complete thermoelastic loading is considered. The solid is $5 \mathrm{~mm}$ high and $10 \mathrm{~mm}$ long. Its properties are: $E_{\mathrm{s}}=200 \mathrm{GPa}, v_{\mathrm{s}}=0.3, \alpha_{\mathrm{s}}=12 \times 10^{-6}$ and $k_{\mathrm{s}}=45 \mathrm{~W} / \mathrm{m} / \mathrm{K}$. A thermal heat flux is applied at the center of the free surface over a patch of half width $a=0.2 \mathrm{~mm}$. The distribution is elliptic with a peak value $q_{m}=10^{-7} \mathrm{~W} / \mathrm{m}^{2}$. The other sides of the solid are mechanically clamped and kept at a prescribed temperature.

The effect of a thin coating with similar elastic properties but a different expansion coefficient is studied. $\alpha_{c}$ is a stepwise function of depth with an abrupt variation across a $1 \mu \mathrm{m}$ thick diffuse boundary. Figs. 11 and 12 show the variation of the maximum value of $\sigma_{y y}$ (in the reference frame defined by Fig. 2) and the Von Mises stress $\sigma_{\mathrm{vm}}$ values as a function of the $\alpha_{\mathrm{c}} / \alpha_{\mathrm{s}}$ ratio. The normal stress component $\sigma_{x x}$ and the shear component $\sigma_{x y}$, being very small, have not been plotted. The Von Mises stress is defined as follows:

$\sqrt{\frac{1}{6}\left[\left(\sigma_{x x}-\sigma_{y y}\right)^{2}+\left(\sigma_{x x}-\sigma_{z z}\right)^{2}+\left(\sigma_{y y}-\sigma_{z z}\right)^{2}\right]+\sigma_{x y}^{2}}$.

The $\sigma_{y y}$ and $\sigma_{\mathrm{vm}}$ stresses have also been computed inside the solid. The peak values are located at the free surface. They have been compared to the values obtained by Leroy [2] with a model based on discrete layers with sharp changing properties. The results are in a good agreement.

\subsection{Graded properties}

FGMs are anisotropic materials, in which the thermomechanical properties vary continuously with depth. In this manner, the sharp mismatch is eliminated, resulting in an

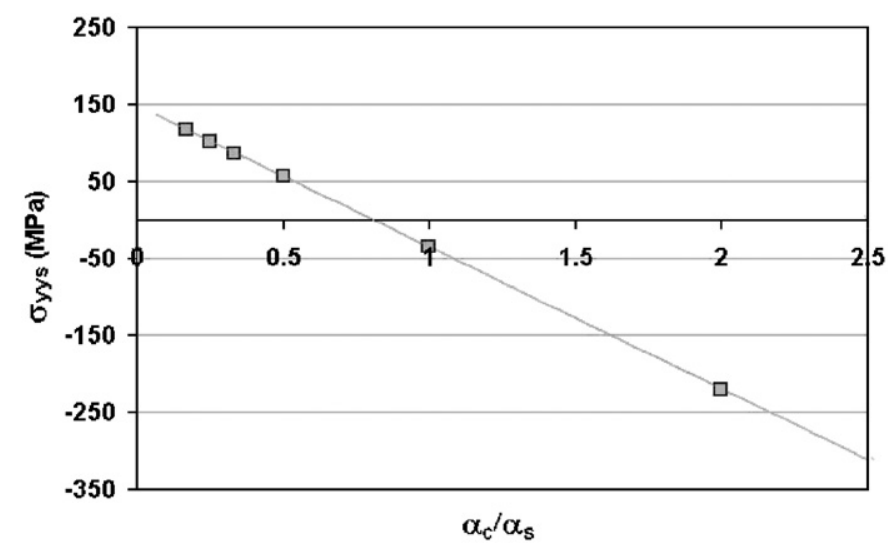

Fig. 11. $\sigma_{y y}$ peak stresses at the surface as a function of $\alpha_{c} / \alpha_{s}$, $H_{\mathrm{c}}=10 \mu \mathrm{m}$. 


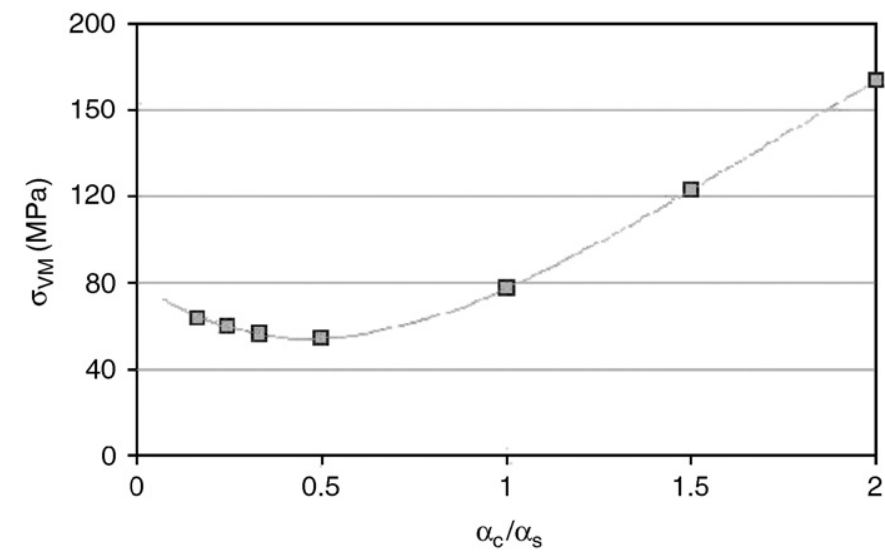

Fig. 12. $\sigma_{\mathrm{vm}}$ peak stresses at the surface as a function of $\alpha_{\mathrm{c}} / \alpha_{\mathrm{s}}$, $H_{\mathrm{c}}=10 \mu \mathrm{m}$.

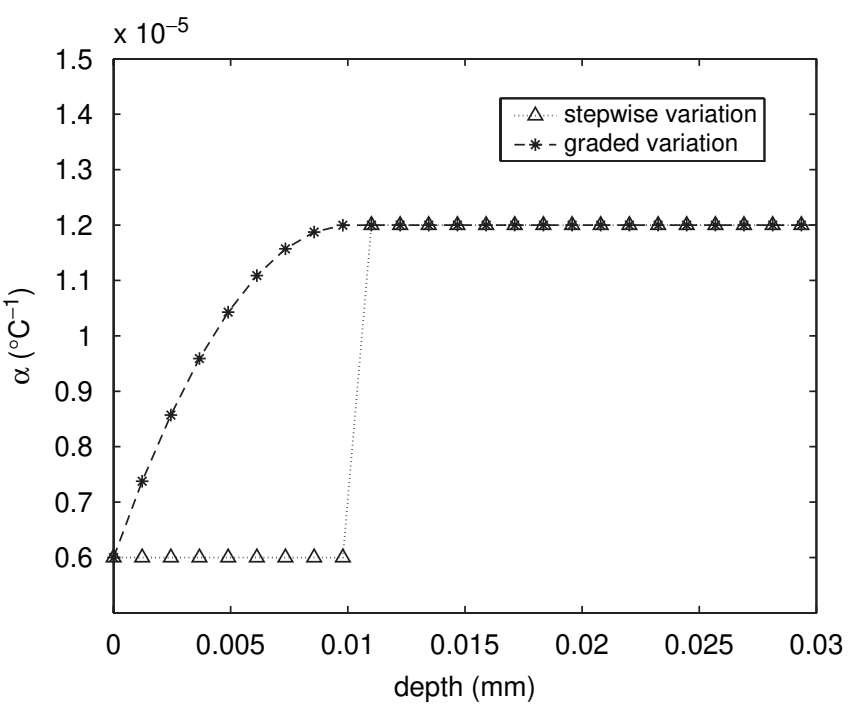

Fig. 13. Stepwise and graded properties.

reduction in stress discontinuities and stress concentrations computed numerically. The effect of a graded variation of the thermal expansion coefficient $(\alpha)$ is analyzed and compared to those of a stepwise change. The variation of $\alpha$ is presented in Fig. 13.

The effect of such a gradation on the stresses is shown in Figs. 14 and 15. The discontinuity at the interface vanishes but the maximum stresses in the coating and in the substrate are almost constant.

\section{Conclusion and further research}

A 2D thermoelastic model for heterogeneous materials is presented. It is based on the Navier and Fourier equations in non-homogeneous media. This model can handle thin layers using multigrid techniques which enable fine mesh sizes and local refinement methods. These methods keep calculation time acceptable on desktop computers.

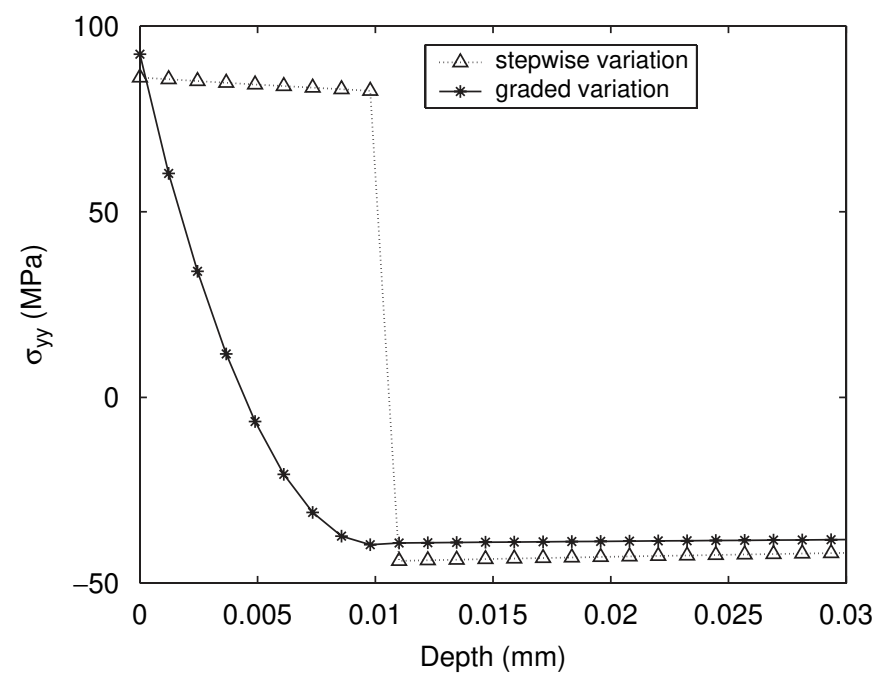

Fig. 14. $\sigma_{y y}$ in the center of the solid, $H_{\mathrm{c}}=10 \mu \mathrm{m}$.

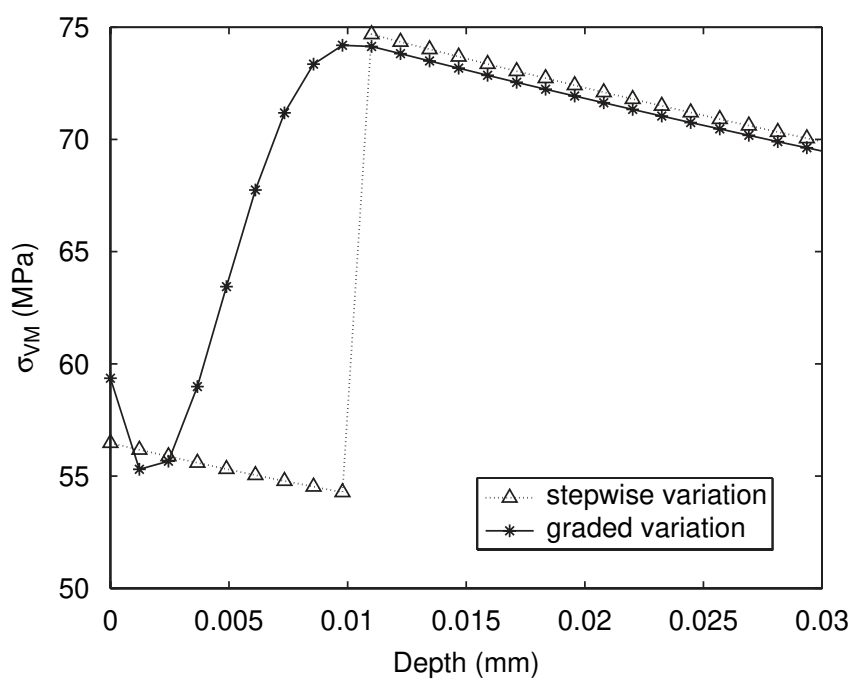

Fig. 15. $\sigma_{\mathrm{vm}}$ in the center of the solid, $H_{\mathrm{c}}=10 \mu \mathrm{m}$.

Moreover, thin coatings do not have constant properties. The deposition process and the physical behavior of the material generate properties that vary from the substrate to the coating bulk value. This model can represent these phenomena with any type of property variation as shown in the last section. It has been shown that graded variation avoids the discontinuity in stress components. Only the expansion coefficient has been studied, but other mechanical and thermal coefficients can also be modified. The main objective of this model is to study and implement numerical techniques before the extension towards 3D thermoelasticity. Multigrid techniques and local refinement can reduce the calculation time. Hence, parametric studies can be conducted with this model. Further extensions will be towards non-linear behavior and solution of the contact problem. 


\section{References}

[1] Ju Y, Liu S. Parameters affecting thermomechanical cracking in coated media due to high speed friction load. J Tribol Trans ASME 1988;110:222-7.

[2] Leroy JM, Floquet A, Villechaise B. Thermomecanical behavior of multilayered media: theory. J Tribol Trans. ASME 1989;111:538-44.

[3] Plumet S, Baietto-Dubourg MC. A 3D model for a multilayered body loaded normally and tangentially against a rigid body: application to specific coatings. J Tribol Trans ASME 1998;120:668-76.

[4] Polonsky IA, Keer LM. A fast and accurate method for numerical analysis of elastic layered contacts. J Tribol Trans ASME 2000; 122:30-5.

[5] Polonsky IA, Keer LM. Fast method for solving rough contact problems: a comparative study. J Tribol Trans ASME 2000;122:36-41.

[6] Brandt A, Lubrecht AA. Multilevel matrix multiplication and fast solution of integral equations. J Comput Phys 1990;90:348-70.

[7] Nogi T, Kato T. Influence of a hard surface layer on the limit of elastic contact: part 1: analysis using a real surface model. J Tribol Trans ASME 1997;119:493-500.

[8] Liu S, Wang Q, Liu G. A versatile method of discrete convolution and FFT (DC-FFT) for contact analysis. Wear 2000;243:101-11.

[9] Liu S, Wang Q. Studying contact stress fields caused by surface tractions with a discrete convolution and fast Fourier transform algorithm. J Tribol Trans ASME 2000;124:36-45.
[10] Bayada G, Chambat M, Lhalouany K. Asymptotic analysis of a thinlayer device with Tresca's contact law in elasticity. Math Methods Appl Sci 1999;22:811-36.

[11] Ye N, Komvopoulos K. Three-dimensional finite element analysis of elastic-plastic layered media under thermomechanical surface loading. J Tribol Trans ASME 2003;125:52-9.

[12] Ozisik MN. Heat conduction. New York: Wiley; 1976 ISBN 0-47105481-X. p. 687.

[13] Saada AS. Elasticity: theory and applications. Robert E. Krieger Publishing Company; 1983 ISBN 0-89874-559-4. p. 643.

[14] Brandt A. Multigrid techniques: 1984 guide with applications to fluid dynamics. Gesellschaft fur Mathematik und Datenverarbeitung MBH BOHN; 1984. p. 176.

[15] Brandt A. Multilevel adaptative solution to boundary value problems. Math Comput 1977;31:333-90.

[16] Yavneh I, Venner CH, Brandt A. Fast multigrid solution of the advection problem with closed characteristics. SIAM J Sci Comput 1998;19:111-25.

[17] Venner C, Lubrecht AA. MultiLevel methods in lubrication. Amsterdam: Elsevier; 2000 ISBN 0-444-50503-2. p. 379.

[18] Brandt A, Israeli M, Yavneh I, Siegel A. Multigrid solution of an elliptic boundary-value problem with integral constraints. SIAM J Sci Comput 2000;21:1357-69.

[19] Bai D, Brandt A. Local mesh refinement multilevel techniques. SIAM J Sci Stat Comput 1987;8:109-34. 